Short communication

\title{
$\alpha$-maxmin solutions to fair division problems and the structure of the set of Pareto utility profiles
}

\author{
F. Hüsseinov* \\ Department of Economics, Bilkent University, 06800 Bilkent, Ankara, Turkey
}

\section{A R T I C L E I N F O}

Article history:

Received 10 July 2008

Received in revised form

29 September 2008

Accepted 17 November 2008

Available online 25 November 2008

\begin{abstract}
A B S T R A C T
A simple proof of the equivalence of Pareto optimality plus positiveness and $\alpha$-maxmin optimality, dispensing with the assumption of closedness of the utility possibility set, is given. Also the structure of the set of Pareto optimal utility profiles is studied.
\end{abstract}

(C) 2008 Elsevier B.V. All rights reserved.

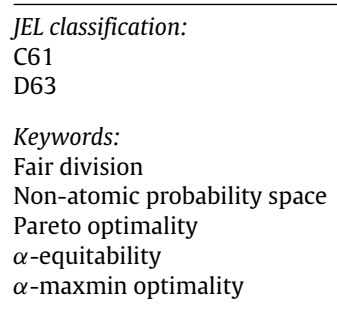

The main results of the recent paper by Sagara "A characterization of $\alpha$-maxmin solutions of fair division problems" published in Mathematical Social Sciences 55 (2008), 273-280, are concerned with the important issue of fair and efficient division of a measurable space among finitely many individuals. One of these results is concerned with the equivalence of Pareto optimality plus positiveness and $\alpha$-maxmin optimality, and assumes the closedness of the utility possibility set. We suggest here a simple proof of this equivalence that dispenses with the closedness assumption. Sagara studies also the structure of the Pareto optimal utility profiles' set, UP. He shows that if the utility possibility set, $U$, is closed then $U P$ is homeomorphic with the standard closed simplex, $\Delta$, in $R^{n}$. Here $n$ is the number of individuals. We give here a short proof of this result and make further observations on the structure of sets $U P$ and $U$.

\footnotetext{
* Tel.: +90 312266 4216; fax: +90 3122665140 .

E-mail address: farhad@bilkent.edu.tr.
} 
First we recall some definitions. A partition scheme of a measurable space $(\Omega, \mathcal{F})$ consists of the non-atomic probability measures $\mu_{1}, \ldots, \mu_{n}$ on this space and the set functions $f_{1}, \ldots, f_{n}$ that map $\mathcal{F}$ into $[0,1]$, such that $f_{i}(\emptyset)=0$ and $f_{i}(\Omega)=1$, for every $i \in N=\{1, \ldots, n\}$.

A partition of $\Omega$ is an ordered $n$-tuple of disjoint sets in $\mathcal{F}$ whose union is $\Omega$. Denote by $\mathcal{P}$ the set of all partitions of $\Omega$. A partition $\left(A_{1}, \ldots, A_{n}\right)$ is positive if $\mu_{i}\left(A_{i}\right)>0$ for all $i \in N$.

A partition $\left(A_{1}, \ldots, A_{n}\right)$ is $\alpha$-maxmin optimal (for $\alpha \in \stackrel{\Delta}{-}$-the interior of $\Delta$ ) if it solves the problem: $\max \left\{\min _{i \in N} \alpha_{i}^{-1} f_{i}\left(A_{i}\right) \mid\left(A_{1}, \ldots, A_{n}\right) \in \mathcal{P}\right\}$. A partition $\left(A_{1}, \ldots, A_{n}\right)$ is $\alpha$-equitable if $\alpha_{i}^{-1} f_{i}\left(A_{i}\right)=$ $\alpha_{j}^{-1} f_{j}\left(A_{j}\right)$ for all $i, j \in N$.

A set function $f_{i}$ is:

(a) $\mu_{i}$-continuous from below if $B_{1} \subset B_{2} \subset \cdots \subset B$ and $\mu_{i}\left(B \backslash \cup B_{k}\right)=0$ imply $f_{i}\left(B_{k}\right) \rightarrow f_{i}(B)$,

(b) strictly $\mu_{i}$-monotone if $A \subset B$ and $\mu_{i}(A)<\mu_{i}(B)$ imply $f_{i}(A)<f_{i}(B)$.

Property (a) is equivalent to the following two properties: $B_{1} \subset B_{2} \subset \cdots$ imply $f_{i}\left(B_{k}\right) \rightarrow f_{i}\left(\cup B_{k}\right)$, and for $A, B \in \mathcal{F}, \mu_{i}(A \Delta B)=0$, where $A \Delta B=(A \cup B) \backslash(A \cap B)$, implies $f_{i}(A)=f_{i}(B)$.

Here $f_{i}(A)$ is the worth of set $A$ to player $i$ and measures $\mu_{i}$ are technical tools that are used to define possible utilities.

The utility possibility set is defined as

$$
U=\left\{\left(x_{1}, \ldots, x_{n}\right) \in[0,1]^{n}: \exists\left(A_{1}, \ldots, A_{n}\right) \in \mathcal{P} \text { such that } x_{i} \leq f_{i}\left(A_{i}\right), i \in N\right\} .
$$

The set of all Pareto optimal utility profiles is denoted as UP.

The Pareto optimal and the weak Pareto optimal partitions and utility profiles are defined as usual through the concepts of Pareto improvement and strict Pareto improvement. When measures $\mu_{1}, \ldots, \mu_{n}$ are absolutely continuous with respect to each other and the assumptions (a) and (b) above are satisfied, a standard argument used in the classical exchange models shows that the notions of weak and strong Pareto optimality coincide (Lemma 4.1 in Sagara's work).

Theorem. Let $\left\{(\Omega, \mathcal{F}), \mu_{i}, f_{i}, i \in N\right\}$ be a partition scheme such that $f_{i}$ is $\mu_{i}$-continuous from below and strictly $\mu_{i}$-monotone for each $i \in N$, and let measures $\mu_{1}, \ldots, \mu_{n}$ be absolutely continuous with respect to each other. Then the following hold.

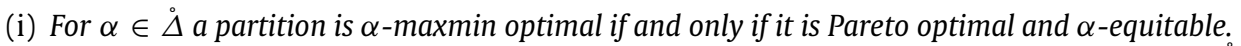

(ii) A partition is Pareto optimal and positive if and only if it is $\alpha$-maxmin optimal for some $\alpha \in \dot{\Delta}$.

Proof. Part (i) is proved in Sagara (2008). We prove (ii). Let partition $P=\left(A_{1}, \ldots, A_{n}\right)$ be Pareto optimal and positive. Setting $\alpha_{i}=\frac{\sum_{k \in N}\left[f_{k}\left(A_{k}\right)\right]^{-1}}{\left[f_{i}\left(A_{i}\right)\right]^{-1}}$ for $i \in N$, we will have $\alpha \in \stackrel{\circ}{\Delta}$ and

$$
\alpha_{i}^{-1} f_{i}\left(A_{i}\right)=\alpha_{j}^{-1} f_{j}\left(A_{j}\right) \text { for all } i, j \in N \text {. }
$$

Now $P$ is Pareto optimal and $\alpha$-equitable for $\alpha \in \dot{\Delta}$. By part (i) $P$ is $\alpha$-maxmin optimal.

Conversely, let partition $P=\left(A_{1}, \ldots, A_{n}\right)$ be $\alpha$-maxmin optimal for $\alpha \in \stackrel{\Delta}{\Delta}$. By part (i) then $P$ is Pareto optimal and $\alpha$-equitable, that is Eq. (1) are satisfied. Now if $f_{j}\left(A_{j}\right)=0$ for some $j \in N$, it follows from Eq. (1) that $f_{i}\left(A_{i}\right)=0$ for all $i \in N$. From properties of functions $f_{i}$ it follows that $\mu_{i}\left(A_{i}\right)=0$. By absolute continuity of measures $\mu_{i}, i \in N$ we have $\mu_{i}\left(A_{j}\right)=0$ for all $i, j \in N$. Hence $\mu_{1}(\Omega)=0$, which contradicts the assumption $\mu_{1}(\Omega)=1$.

Remark. Obviously, for any measures on $(\Omega, \mathcal{F})$ which are absolutely continuous with respect to each other each of properties (a) and (b) is satisfied for one of them if and only if it is satisfied for the other. Therefore the assumption of absolute continuity of measures with respect to each other made in the theorem simplifies the partition scheme to $\left\{(\Omega, \mathcal{F}, \mu), f_{i}, i \in N\right\}$, where, for example, $\mu=\mu_{1}$.

In the remaining part of this note we study the structure of the utility possibility set, $U$, and Pareto optimal utility profiles' set, UP. We start with giving a short proof of Sagara's Theorem 4.1 concerned with the structure of $U P$.

For vectors $x, y \in R^{n}$ we will write $x>y$ if $x_{i}>y_{i}$ for all $i \in N$. Define a mapping $h: \Delta \rightarrow$ $R^{n}$ as $h(x)=\rho(x) x$ for $x \in \Delta$, where $\rho$ is defined as $\rho(x)=\sup \{r \geq 0 \mid r x \in U\}$.

Further we will assume that all assumptions of the above theorem are satisfied. 
Proposition 1. If $U$ is closed, then $h$ is a homeomorphism between $\Delta$ and UP.

Proof. Since $U$ is closed, $h(x) \in U$. Since a weak Pareto optimal utility profile is Pareto optimal $h(x) \notin U P$ would imply that there exists $u \in U$ such that $u>h(x)$. Then by comprehensiveness of $U, h(x)$ would be a relative interior point of $U$, which contradicts its definition. Since for each $x \in U \backslash U P$ there exists $y \in U$ such that $y>x$, it follows that $U \backslash U P$ is a relative open subset of $[0,1]^{n}$, and hence UP is a closed set. Since measures $\mu_{1}, \ldots, \mu_{n}$ are absolutely continuous with respect to each other $U$ contains a strictly positive vector. Hence $\rho(x)>0$ for all $x \in \Delta$. It follows easily from this that $h$ is one-to-one. Since $\Delta$ is compact to complete the proof it suffices to show that $h$ is continuous. This will follow if we show that $\rho$ is a continuous function.

Upper semicontinuity of $\rho$. Assume $x_{k} \in \Delta, x_{k} \rightarrow x$ and $\overline{\lim } \rho\left(x_{k}\right)>\rho(x)$. Since $\rho$ is bounded there exists a subsequence $\left\{y_{k}\right\}$ of sequence $\left\{x_{k}\right\}$ such that $\rho\left(y_{k}\right) \rightarrow \rho_{0}>\rho(x)$. Closedness of $U$ implies that $\rho_{0} x \in U$. But $\rho_{0}>\rho(x)$ then would imply that $h(x)=\rho(x) x$ is not a Pareto optimal utility profile. This contradicts the definition of function $h$.

Lower semicontinuity of $\rho$. Assume $x_{k} \in \Delta, x_{k} \rightarrow x$ and $\underline{\lim } \rho\left(x_{k}\right)<\rho(x)$. Then there exists a subsequence $\left\{z_{k}\right\}$ of sequence $\left\{x_{k}\right\}$, such that $\rho\left(z_{k}\right) \rightarrow \rho_{1}<\rho(x)$. Thus $\left\{\rho\left(z_{k}\right) z_{k}\right\}$ is a sequence in $U P$ with the limit $\rho_{1} x$ not in UP. This contradicts the closedness of UP.

Next we show that the utility possibility set $U$ is homeomorphic to $D=\left\{x \in R^{n}: x_{i} \geq 0, i \in\right.$ $N$ and $|x| \leq 1\}$, where $|x|=x_{1}+\cdots+x_{n}$, and hence to the standard closed simplex in $R^{n}$.

Proposition 2. If $U$ is closed then it is homeomorphic to $D$.

Proof. By Proposition 1 mapping $h: \Delta \rightarrow U P$ is a homeomorphism. Define a mapping $H: U \rightarrow D$ by setting

$$
H(x)= \begin{cases}\frac{x}{\left|h\left(\frac{x}{|x|}\right)\right|} & \text { for } x \in U \backslash\{0\}, \\ 0 & \text { for } x=0\end{cases}
$$

If $x, y \in U \backslash\{0\}$ are proportional and $x \neq y$, then $\left|h\left(\frac{x}{|x|}\right)\right|=\left|h\left(\frac{y}{|y|}\right)\right|$ and hence $H(x) \neq H(y)$. Also if $x \in U \backslash\{0\}$ then $H(x) \neq H(0)$. So $H$ is one-to-one. Since the distance between 0 and UP is positive it follows that $H$ is continuous. Since $U$ is compact it follows that $H$ is a homeomorphism.

Denote $U_{i}=U \cap\left\{x \in R^{n}: x_{i}=0\right\}, \forall i \in N$. Let $\Pi_{i}^{\prime}$ be the orthogonal projection map of $R^{N}$ into $R^{N_{-i}}$, where $N_{-i}=N \backslash\{i\}$, and let $\Pi_{i}$ be its restriction into UP.

Proposition 3. If $U$ is closed then for each $i \in N$ map $\Pi_{i}$ is a homeomorphism.

Proof. Let $v$ be an arbitrary point in $U_{i}$. Then since $U$ is compact the intersection $U \cap\left\{x \in R^{n}: \Pi_{i}^{\prime}(x)=\right.$ $v$ \} is a compact set. Hence there is a point $u$ in this set that has the largest $i$ th component. We assert $u \in U P$. If not, then since a non-Pareto optimal utility profile is not weakly Pareto optimal, $u$ is not weakly Pareto optimal. Thus there exists a point $u^{\prime} \in U$ such that $u^{\prime}>u$. Since $U$ is comprehensive it follows that there is a point $u^{\prime \prime} \in U$ with $\Pi_{i}^{\prime}\left(u^{\prime \prime}\right)=v$ and $u^{\prime \prime}>u$. This contradicts to the choice of $u$. So $\Pi_{i}$ is an onto map.

Now if $\Pi_{i}(u)=\Pi_{i}\left(u^{\prime}\right)$, that is $u_{-i}=u_{-i}^{\prime}$ for $u, u^{\prime} \in U P$ then $u_{i}=u_{i}^{\prime}$; otherwise one of the profiles $u, u^{\prime}$ would not be Pareto optimal. So $\Pi_{i}$ is a one-to-one map. Since $\Pi_{i}$ is continuous and UP is compact, $\Pi_{i}$ is a homeomorphism.

\section{Acknowledgements}

I am grateful to Özgür Evren for useful discussions and an anonymous referee for comments that led to a number of improvements. 